Erasmus MC University Medical Center Rotterdam rapung

\title{
Comparison of three read-out methods for Sensititre YeastOne Alamar blue assay for determining Minimum Inhibitory Concentrations
}

Bertrand Nyuykonge ${ }^{1}$, Ahmed Fahal ${ }^{2}$, Peter D Croughs ${ }^{1}$, Wendy W.J Van de Sande ${ }^{1}$

'Erasmus MC, Department of Medical Microbiology and Infectious Disease, Rotterdam, The Netherlands, w.vandesande@erasmusmc.nl

2Mycetoma Research Center, Khartoum, Sudan

\section{Introduction}

Madurella mycetomatis (M.mycetomatis) is the major causative agent of eumycetoma. Eumycetoma is a neglected, inflammatory tropical infection characterized by painless lesions, grains and draining sinuses. Currently, susceptibility testing for $M$. mycetomatis is not routinely performed. This is possibly due to the need to use hyphal suspension as a starting material, the slow growth rate, labour-intensiveness and the need to use expensive dyes for endpoint visualization. Furthermore, pyomelanin is produced by several of the isolates, which might hamper the use of other viability dyes in in vitro susceptibility testing. Commercial systems such as the Sensititre YeastOne YO10 are available but are currently not used in clinical practice with regards to eumycetoma.

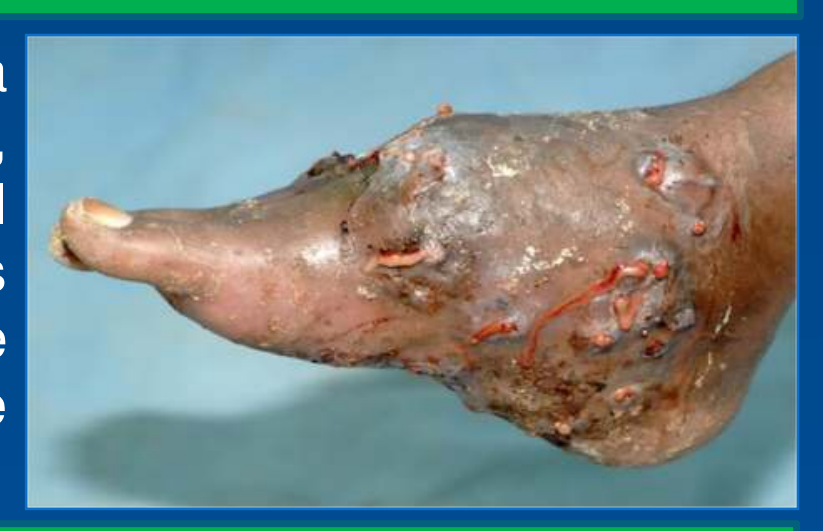

\section{Objectives}

- Determine if the Sensititre YeastOne Y010 Alamar blue assay can be used to determine the in vitro susceptibility of $M$. mycetomatis towards several antifungal agents.

- Assess if pyomelanin formation interferes with the various endpoint read-out systems, by comparing isolates secreting pyomelanin with isolates which do not secrete pyomelanin

\section{Materials \& Methods}

- Eight pyomelanin producing (P1, MM25, MM30, MM44, MM45, MM49, MM55, MM68) and two non-pyomelanin producing (MM14 and MM50) M. mycetomatis isolates were used in this study.

- In vitro susceptibilities of 10 clinical isolates of $M$. mycetomatis were determined with the Sensititre YeastOne Alamar blue susceptibility assay following manufacturer's instructions. The Minimum Inhibitory concentrations, (MICs) were determined by visual reading, SWIN reading and spectrophotometry.

\section{Results}

- M. mycetomatis is most susceptible towards the azole class of antifungal agents

- Different MICs were obtained with different read-outs system

- Better agreement between visual and SWIN endpoints compared to visual and spectrophotometer and/or SWIN

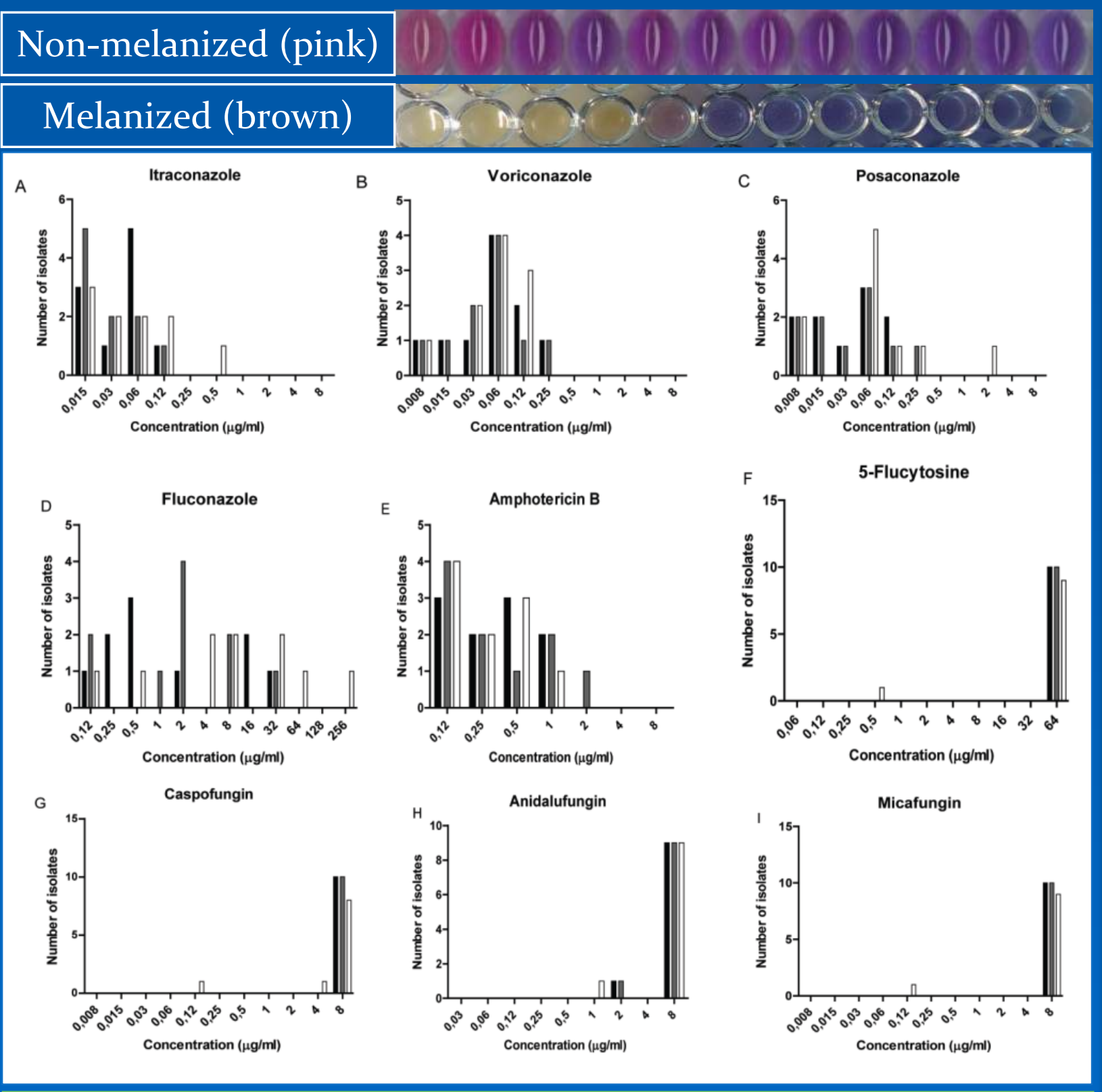

Figure 1: Sensititre YeastOne panel (non-melanized pink and melanized, brown). MICs distribution for isolates of M. mycetomatis with three different read-out methods, black bars:visual, gray bars: SWIN and white bars: spectrophotometric

\begin{tabular}{|c|c|c|c|c|c|c|}
\hline \multicolumn{7}{|c|}{$\begin{array}{l}\text { Table 1: Comparison of the different endpoint read-out methods for Sensititre YeastONE } \\
\text { assay for isolates of M.mycetomatis }\end{array}$} \\
\hline \multirow{3}{*}{$\begin{array}{l}\text { Antifungal } \\
\text { agent }\end{array}$} & \multicolumn{6}{|c|}{ Agreement (\%) } \\
\hline & \multicolumn{2}{|c|}{ Visual vs SWIN } & \multicolumn{2}{|c|}{$\begin{array}{c}\text { Visual vs } \\
\text { Spectrophotometric }\end{array}$} & \multicolumn{2}{|c|}{$\begin{array}{c}\text { SWIN vs } \\
\text { Spectrophotometric }\end{array}$} \\
\hline & $\begin{array}{l}\text { Identical } \\
\text { MICs }\end{array}$ & $\begin{array}{c}\text { MICs } \\
\text { within } \\
1 \\
\text { dilution }\end{array}$ & $\begin{array}{l}\text { Identical } \\
\text { MICs }\end{array}$ & $\begin{array}{c}\text { MICs } \\
\text { within } 1 \\
\text { dilution }\end{array}$ & $\begin{array}{l}\text { Identical } \\
\text { MICs }\end{array}$ & $\begin{array}{c}\text { MICs } \\
\text { within } 1 \\
\text { dilution }\end{array}$ \\
\hline Itraconazole & 80 & 100 & 50 & 70 & 50 & 80 \\
\hline Vorinacozole & 80 & 100 & 40 & 80 & 30 & 70 \\
\hline Posaconazole & 90 & 100 & 40 & 80 & 50 & 80 \\
\hline Fluconazole & 30 & 70 & 0 & 30 & 20 & 50 \\
\hline $\begin{array}{c}\text { Amphotericin } \\
\text { B }\end{array}$ & 70 & 100 & 50 & 80 & 30 & 80 \\
\hline 5-Flucytosine & 100 & 100 & 90 & 90 & 90 & 90 \\
\hline Caspofungin & 100 & 100 & 80 & 90 & 80 & 90 \\
\hline Micafungin & 100 & 100 & 90 & 90 & 90 & 90 \\
\hline Anidalufungin & 100 & 100 & 90 & 100 & 90 & 100 \\
\hline
\end{tabular}

\section{Conclusion}

The present data suggest the Sensititre YeastOne YO10 is suitable for the susceptibility testing of M. mycetomatis and could vital in resourcelimited setting

M. mycetomatis isolates are susceptible to azoles and amphotericin B but not echinocandins and 5-flucytosine 